ITP-UH-01/08

\title{
Non-Abelian Vortices on Riemann Surfaces: an Integrable Case*
}

\author{
Alexander D. Popov \\ Institut für Theoretische Physik, Leibniz Universität Hannover \\ Appelstraße 2, 30167 Hannover, Germany \\ Email: popov@itp.uni-hannover.de \\ and \\ Bogoliubov Laboratory of Theoretical Physics, JINR \\ 141980 Dubna, Moscow Region, Russia \\ Email: popov@theor.jinr.ru
}

\begin{abstract}
We consider $\mathrm{U}(n+1)$ Yang-Mills instantons on the space $\Sigma \times S^{2}$, where $\Sigma$ is a compact Riemann surface of genus $g$. Using an $\mathrm{SU}(2)$-equivariant dimensional reduction, we show that the $\mathrm{U}(n+1)$ instanton equations on $\Sigma \times S^{2}$ are equivalent to non-Abelian vortex equations on $\Sigma$. Solutions to these equations are given by pairs $(A, \phi)$, where $A$ is a gauge potential of the group $\mathrm{U}(n)$ and $\phi$ is a Higgs field in the fundamental representation of the group $\mathrm{U}(n)$. We briefly compare this model with other non-Abelian Higgs models considered recently. Afterwards we show that for $g>1$, when $\Sigma \times S^{2}$ becomes a gravitational instanton, the non-Abelian vortex equations are the compatibility conditions of two linear equations (Lax pair) and therefore the standard methods of integrable systems can be applied for constructing their solutions.
\end{abstract}

* Supported in part by the Deutsche Forschungsgemeinschaft. 


\section{Introduction and summary}

The Abelian Higgs model on $\mathbb{R} \times \mathbb{R}^{2}$ in the Bogomolny regime admits static vortex solutions [1] which describe magnetic flux tubes (vortex strings) penetrating a two-dimensional superconductor. It is widely believed that (electric) vortex strings play an important role in the confinement of quarks. Many results known for the Abelian Higgs model were generalized to compact Riemann surfaces and to the non-Abelian case (see e.g. [2]-[12] and references therein). These generalizations are of interest for many reasons. One reason for having vortices on a compact Riemann surface $\Sigma$ is to control the thermodynamic limit $N \rightarrow \infty$ with the density of vortices $N /$ area of $\Sigma$ \} fixed [3, 4]. In this case one can reduce the evaluation of the partition function of the gas of vortices to the computation of the volume of the moduli space $[3,4]$. Note that the moduli space of vortices on Riemann surfaces is compact (contrary to the case $\Sigma=\mathbb{R}^{2}$ ) and it naturally appears in the description of Gromov-Witten and other topological invariants (see e.g. [6, 13]). Also, vortices on $\Sigma$ and corresponding Yang-Mills instantons on $\Sigma \times S^{2}$ appear [12] in recently proposed twistor string theory $[14,15]$. On the other hand, non-Abelian vortices are actively studied presently since the confinement of quarks can be related with a condensation of non-Abelian monopoles and a non-Abelian analog of dual Meissner effect (see e.g. [9, 10, 11]).

In this note, we consider $\mathrm{U}(n)$ Yang-Mills-Higgs theory on a Riemann surface $\Sigma$ with a Higgs field in the fundamental representation of the group $\mathrm{U}(n)$. Contrary to the flat space $\mathbb{R}^{2}$, where such theory reduces to the Abelian one, on Riemann surfaces even the simplest model with one Higgs field (one flavour) is really non-Abelian. Experience with other models shows that it is useful to know solutions exactly - this can facilitate the analysis of their properties, their moduli space etc. In fact, there exist well-developed solution-generating techniques for so-called integrable models. We show that the vortex equations on $\Sigma$ are integrable under certain conditions. Namely, it is shown that non-Abelian vortices on $\Sigma$ can be identified with $\mathrm{SO}(3)$-symmetric Yang-Mills instantons on $\Sigma \times S^{2}$. Furthermore, for the genus $g>1$ one can choose a metric on $M=\Sigma \times S^{2}$ such that the scalar curvature of $M$ vanishes and this manifold becomes [16] a (conformal) gravitational instanton [17, 18]. Then the Yang-Mills instanton equations on $\Sigma \times S^{2}$ become integrable as well as the non-Abelian vortex equations on $\Sigma$. We describe geometrical and topological conditions for their integrability and write down the explicit Lax pair for the vortex equations.

\section{$2 \quad$ Manifolds $\Sigma \times \mathbb{C} P^{1}$}

Here we consider Kähler metrics on the product $\Sigma \times \mathbb{C} P^{1}$ of a real two-dimensional manifold $\Sigma$ and the Riemann sphere $\mathbb{C} P^{1}$. As $\Sigma$ we will take the Euclidean space $\mathbb{R}^{2}$ or a compact Riemann surface of genus $g \geq 0$. The case of $\mathbb{R}^{2}$ is kept for the Abelian subcase.

Riemann surfaces. For a Riemann surface $\Sigma$, the metric and the volume form are given in local (conformal) coordinates $z, \bar{z}$ by

$$
\mathrm{d} s_{\Sigma}^{2}=2 g_{z \bar{z}} \mathrm{~d} z \mathrm{~d} \bar{z} \quad \text { and } \quad \omega_{\Sigma}=\mathrm{i} g_{z \bar{z}} \mathrm{~d} z \wedge \mathrm{d} \bar{z}
$$

respectively. Furthermore, for the nonvanishing components of the Christoffel symbol and the Ricci tensor we have

$$
\begin{aligned}
& \Gamma_{z z}^{z}=2 \partial_{z} \log \rho \quad \text { and } \quad \Gamma_{\bar{z} \bar{z}}^{\bar{z}}=2 \partial_{\bar{z}} \log \rho \quad \text { with } \rho^{2}:=g_{z \bar{z}} \\
& R_{z \bar{z}}=-2 \partial_{z} \partial_{\bar{z}} \log \rho=\varkappa g_{z \bar{z}} \quad \Longrightarrow \quad R_{\Sigma}=2 g^{z \bar{z}} R_{z \bar{z}}=2 \varkappa
\end{aligned}
$$


where $R_{\Sigma}$ is the (constant) scalar curvature of $\Sigma$. In real local coordinates $x^{1}, x^{2}$ on $\Sigma$ we have $z=x^{1}+\mathrm{i} x^{2}$ and $\bar{z}=x^{1}-\mathrm{i} x^{2}$. For $\mathbb{R}^{2}$ one should simply put $g_{z \bar{z}}=\delta_{z \bar{z}}, R_{z \bar{z}}=0$ and $\varkappa=0$.

Monopole bundle over $\mathbb{C} P^{1}$. Formulae (2.1)-(2.3) for the standard two-sphere $S^{2} \cong \mathbb{C} P^{1}$ of constant radius $R$ read

$$
\begin{gathered}
\mathrm{d} s_{\mathbb{C} P^{1}}^{2}=\frac{4 R^{4}}{\left(R^{2}+y \bar{y}\right)^{2}} \mathrm{~d} y \mathrm{~d} \bar{y} \quad \text { and } \quad \omega_{\mathbb{C} P^{1}}=-\frac{2 \mathrm{i} R^{4}}{\left(R^{2}+y \bar{y}\right)^{2}} \mathrm{~d} y \wedge \mathrm{d} \bar{y}, \\
R_{y \bar{y}}=-\partial_{y} \partial_{\bar{y}} \log \tilde{\rho}^{2}=\tilde{\varkappa} g_{y \bar{y}}=\frac{1}{R^{2}} \tilde{\rho}^{2} \Rightarrow \quad R_{\mathbb{C} P^{1}}=\frac{2}{R^{2}} \quad \text { and } \quad \tilde{\varkappa}=\frac{1}{R^{2}} .
\end{gathered}
$$

Note that (2.4) corresponds to the choice of orientation on $S^{2}$ inverse to the canonical one, i.e. $y=x^{3}-\mathrm{i} x^{4}$ and $\bar{y}=x^{3}+\mathrm{i} x^{4}$ for real local coordinates $x^{3}, x^{4}$ on $S^{2}$.

Consider now the Hermitian complex line bundle ${ }^{1} \mathcal{L} \rightarrow \mathbb{C} P^{1}$ with a unitary Abelian connection $a$ and the curvature $f$ having the form

$$
a=\frac{1}{2\left(R^{2}+y \bar{y}\right)}(\bar{y} \mathrm{~d} y-y \mathrm{~d} \bar{y}) \quad \text { and } \quad f=\mathrm{d} a=-\frac{R^{2}}{\left(R^{2}+y \bar{y}\right)^{2}} \mathrm{~d} y \wedge \mathrm{d} \bar{y}=\frac{1}{2 \mathrm{i} R^{2}} \omega_{\mathbb{C} P^{1}} .
$$

This is the Dirac monopole bundle over $\mathbb{C} P^{1}$.

Gravitational instantons. For the scalar curvature $R_{M}$ of the manifold $M=\Sigma \times \mathbb{C} P^{1}$ we have $R_{M}=2(\varkappa+\tilde{\varkappa})$. Note that for

$$
\varkappa=-\tilde{\varkappa}=-\frac{1}{R^{2}}
$$

the Kähler manifold $M$ has self-dual Weyl curvature tensor [16]. Such manifolds are considered as gravitational instantons in conformal gravity (see e.g. [17, 18, 20]). At the same time, the manifold $M=\mathbb{C} P^{1} \times \mathbb{C} P^{1}$ with $\varkappa=\tilde{\varkappa}$ is a smooth Einstein manifold which can be considered as a gravitational instanton in Euclidean quantum gravity (see e.g. [21] and references therein).

\section{Non-Abelian vortices on $\Sigma$ as Yang-Mills instantons on $\Sigma \times \mathbb{C} P^{1}$}

SU(2)-equivariant gauge potential. Consider the manifold $M=\Sigma \times \mathbb{C} P^{1}$. Let $\mathcal{E} \rightarrow M$ be an $\mathrm{SU}(2)$-equivariant ${ }^{2}$ complex vector bundle of rank $r \geq 2$ over $M$ with the group $\mathrm{SU}(2)$ acting trivially on $\Sigma$ and in the standard way by $\mathrm{SU}(2)$-isometry on $\mathbb{C} P^{1}=\mathrm{SU}(2) / \mathrm{U}(1)$. Let $\mathcal{A}$ be a $u(r)$-valued local form of $\mathrm{SU}(2)$-equivariant connection on $\mathcal{E}$ (see e.g. [5, 7]); it can be chosen in the form

$$
\mathcal{A}=\left(\begin{array}{cc}
A^{1}+1_{n} \cdot a & \frac{1}{\sqrt{2}} \phi \bar{\beta} \\
-\frac{1}{\sqrt{2}} \phi^{\dagger} \beta & A^{2}-a
\end{array}\right)
$$

where $A^{1}$ and $A^{2}$ are $u(n)$ - and $u(1)$-valued gauge potentials on the rank $n$ and rank one vector bundles $E_{1}$ and $E_{2}$ over $\Sigma$ with $n+1=r, \phi \in \operatorname{Hom}\left(E_{2}, E_{1}\right)$ is a scalar transforming in bifundamental representation $(n, \overline{1})$ of the group $U(n) \times U(1)$ and $\phi^{\dagger}$ is its Hermitian conjugate. In (3.1), $a$ is the one-monopole gauge potential on $\mathbb{C} P^{1}$ given in (2.6) and

$$
\beta:=\frac{\sqrt{2} R^{2} \mathrm{~d} y}{R^{2}+y \bar{y}} \quad \text { and } \quad \bar{\beta}:=\frac{\sqrt{2} R^{2} \mathrm{~d} \bar{y}}{R^{2}+y \bar{y}}
$$

\footnotetext{
${ }^{1}$ For more detailed description with transition function etc. see e.g. [19] and references therein.

${ }^{2}$ This means a generalized SU(2)-invariance, i.e. invariance under space-time transformations up to gauge transformations $[22,23,5]$.
} 
are forms on $\mathbb{C} P^{1}$ of type $(1,0)$ and $(0,1)$ satisfying

$$
\mathrm{d} \bar{\beta}+2 a \wedge \bar{\beta}=0=\mathrm{d} \beta-2 a \wedge \beta \quad \text { and } \quad \beta \wedge \bar{\beta}=\mathrm{i} \omega_{\mathbb{C} P^{1}} .
$$

Fields $A^{1}, A^{2}$ and $\phi$ depend only on coordinates of $\Sigma$ and the Higgs field $\phi$ can be identified with a section $\left(n \times 1\right.$ column) of the bundle $E:=E_{1} \otimes \bar{E}_{2}$.

Symmetric field strength tensor. In local complex coordinates on $\Sigma \times \mathbb{C} P^{1}$ the curvature $\mathcal{F}=\mathrm{d} \mathcal{A}+\mathcal{A} \wedge \mathcal{A}$ for $\mathcal{A}$ of the form (3.1) has the following field strength components:

$$
\begin{aligned}
& \mathcal{F}_{z \bar{z}}=\left(\begin{array}{cc}
F_{z \bar{z}}^{1} & 0 \\
0 & F_{z \bar{z}}^{2}
\end{array}\right), \quad \mathcal{F}_{y \bar{y}}=\left(\begin{array}{cc}
\frac{1}{2} g_{y \bar{y}}\left(\frac{1}{R^{2}} \cdot 1_{n}-\phi \phi^{\dagger}\right) & 0 \\
0 & -\frac{1}{2} g_{y \bar{y}}\left(\frac{1}{R^{2}}-\phi^{\dagger} \phi\right)
\end{array}\right), \\
& \mathcal{F}_{\bar{z} \bar{y}}=\left(\begin{array}{cc}
0 & \frac{\tilde{\rho}}{\sqrt{2}}\left(\partial_{\bar{z}} \phi+A_{\bar{z}}^{1} \phi-A_{\bar{z}}^{2} \phi\right) \\
0 & 0
\end{array}\right)=-\left(\mathcal{F}_{z y}\right)^{\dagger}, \\
& \mathcal{F}_{z \bar{y}}=\left(\begin{array}{cc}
0 & \frac{\tilde{\rho}}{\sqrt{2}}\left(\partial_{z} \phi+A_{z}^{1} \phi-A_{z}^{2} \phi\right) \\
0 & 0
\end{array}\right)=-\left(\mathcal{F}_{\bar{z} y}\right)^{\dagger} .
\end{aligned}
$$

Here $\tilde{\rho}^{2}=g_{y \bar{y}}, F^{1}=F_{z \bar{z}}^{1} \mathrm{~d} z \wedge \mathrm{d} \bar{z}=\mathrm{d} A^{1}+A^{1} \wedge A^{1}$ and $F^{2}=F_{z \bar{z}}^{2} \mathrm{~d} z \wedge \mathrm{d} \bar{z}=\mathrm{d} A^{2}$.

Non-Abelian vortex equations on $\Sigma$. Let us consider the Yang-Mills instanton equations $* \mathcal{F}=\mathcal{F}$ on $M$, where $*$ is the Hodge operator. In local coordinates on $\Sigma \times \mathbb{C} P^{1}$ these equations have the form

$$
\mathcal{F}_{\bar{z} \bar{y}}=0=\left(\mathcal{F}_{z y}\right)^{\dagger} \quad \text { and } \quad g^{z \bar{z}} \mathcal{F}_{z \bar{z}}+g^{y \bar{y}} \mathcal{F}_{y \bar{y}}=0
$$

Substitution of (3.4) into (3.6) shows that the instanton equations (3.6) on $\Sigma \times \mathbb{C} P^{1}$ are equivalent to non-Abelian $\mathrm{BPS}^{3}$ vortex equations $($ cf. $[2,5,7])$ on $\Sigma$ :

$$
\begin{gathered}
\partial_{\bar{z}} \phi+\left(A_{\bar{z}}^{1}-A_{\bar{z}}^{2} \cdot 1_{n}\right) \phi=0 \\
2 F_{z \bar{z}}^{1}=g_{z \bar{z}}\left(\frac{1}{R^{2}} \cdot 1_{n}-\phi \phi^{\dagger}\right) \text { and } 2 F_{z \bar{z}}^{2}=-g_{z \bar{z}}\left(\frac{1}{R^{2}}-\phi^{\dagger} \phi\right) .
\end{gathered}
$$

In the Abelian case $n=1$ and for $A^{1}=-A^{2}=\frac{1}{2} A$ these equations reduce to the standard Abelian BPS vortex equations on $\Sigma$ (see e.g. $[23,2,12]$ ) which can also be considered on $\Sigma=\mathbb{R}^{2}$.

Topological restrictions. Recall that $A^{1}$ and $A^{2}$ are connections on Hermitian vector bundles $E_{1}$ and $E_{2}$ over $\Sigma$ having rank $n$ and 1, respectively. Calculating their first Chern numbers

$$
N_{1}=\operatorname{deg} E_{1}=c_{1}\left(E_{1}\right)=\frac{\mathrm{i}}{2 \pi} \int_{\Sigma} \operatorname{tr} F^{1} \quad \text { and } \quad N_{2}=\operatorname{deg} E_{2}=c_{1}\left(E_{2}\right)=\frac{\mathrm{i}}{2 \pi} \int_{\Sigma} F^{2}
$$

and integrating traces of $(3.7 \mathrm{~b})$ over $\Sigma$, we obtain (cf. [2])

$$
N_{1} \leq \frac{n}{\varkappa R^{2}}(1-g) \quad \text { and } \quad N_{1}+N_{2}=\frac{1}{\varkappa R^{2}}(n-1)(1-g) .
$$

Here $g \neq 1$ and for $g=1$ (torus) one can derive similar (in)equalities. Thus, there are the topological obstructions (3.9) to the existence of solutions to the vortex equations (3.7).

\footnotetext{
${ }^{3}$ Namely, the first order Bogomolny equations [24] at critical value of the coupling constant.
} 
Non-Abelian Higgs models with $n_{f}=1$. Here we want to compare the vortex equations (3.7) with those considered in non-Abelian Higgs models on $\mathbb{R}^{2}$ with $\mathrm{U}\left(n_{c}\right)$ gauge fields and $n_{f}$ scalar fields in the fundamental representation of the group $\mathrm{U}\left(n_{c}\right)$ (see e.g. $[9,10,11]$ and references therein). In the literature, $n_{c}$ is identified with the number of "colours" and $n_{f}$ with the number of "flavours". In the case of (3.7), one has $n_{c}=n \geq 1$ and $n_{f}=1$. It is usually argued [11] that it should be $n_{f} \geq n_{c}$ since there is no non-Abelian vacuum with vanishing gauge fields and constant Higgs fields if $n_{f}<n_{c}$. However, this argument may be true only for $\Sigma=\mathbb{R}^{2}$. On the contrary, on compact Riemann surfaces $\Sigma$ there are no constant sections $\phi$ (besides zero section) of the non-trivial bundle $E=E_{1} \otimes \bar{E}_{2}$ and the vacuum has more complicated structure. Therefore, on Riemann surfaces the case $n_{f}=1$ is really non-Abelian and (3.7) describes the simplest non-Abelian vortex equations.

For more easy comparing of (3.7) with the non-Abelian vortex equations discussed in the literature [11], we rewrite them in the form

$$
\begin{gathered}
\partial_{\bar{z}} \phi+A_{\bar{z}}^{s u(n)} \phi+A_{\bar{z}}^{u(1)} \phi=0 \\
2 g^{z \bar{z}} F_{z \bar{z}}^{s u(n)}=\frac{1}{n} \operatorname{tr}\left(\phi \phi^{\dagger}\right) \cdot 1_{n}-\phi \phi^{\dagger} \quad \text { and } \quad g^{z \bar{z}} F_{z \bar{z}}^{u(1)}=\frac{1}{R^{2}}-\frac{n+1}{2 n} \phi^{\dagger} \phi
\end{gathered}
$$

where

$$
\begin{gathered}
A^{s u(n)}=A^{1}-\frac{1}{n}\left(\operatorname{tr} A^{1}\right) \cdot 1_{n} \quad \text { and } \quad F^{s u(n)}=F^{1}-\frac{1}{n}\left(\operatorname{tr} F^{1}\right) \cdot 1_{n}, \\
A^{u(1)}=\frac{1}{n} \operatorname{tr} A^{1}-A^{2} \quad \text { and } \quad F^{u(1)}=\mathrm{d} A^{u(1)} .
\end{gathered}
$$

One can also introduce the Abelian fields

$$
\tilde{A}^{u(1)}=\frac{1}{n} \operatorname{tr} A^{1}+A^{2} \quad \text { and } \quad \tilde{F}^{u(1)}=\mathrm{d} \tilde{A}^{u(1)},
$$

but for $n>1$ they are not independent since from (3.7) it follows that

$$
g^{z \bar{z}} \tilde{F}_{z \bar{z}}^{u(1)}=\frac{n-1}{n+1}\left(\frac{1}{R^{2}}-g^{z \bar{z}} F_{z \bar{z}}^{u(1)}\right)=\frac{n-1}{2 n} \phi^{\dagger} \phi .
$$

Recall that $A^{s u(n)}+A^{u(1)} \cdot 1_{n}$ is a connection on the rank- $n$ complex vector bundle $E$ and $\phi \in H^{0}(\Sigma, E)$ is a section of this bundle. Accordingly, the topological restrictions (3.9) can be rewritten for (3.10) in the form

$$
\begin{aligned}
& 0 \leq N=\frac{1}{n} N_{1}-N_{2}=\frac{\mathrm{i}}{2 \pi} \int_{\Sigma} F^{u(1)} \leq \frac{2}{\varkappa R^{2}}(1-g), \\
& 0 \leq \tilde{N}=\frac{1}{n} N_{1}+N_{2}=\frac{\mathrm{i}}{2 \pi} \int_{\Sigma} \tilde{F}^{u(1)} \leq \frac{n-1}{n+1} \frac{2}{\varkappa R^{2}}(1-g),
\end{aligned}
$$

plus obvious equation connecting $N$ and $\tilde{N}$. For a given $g \neq 1$ (for tori one can derive similar formulae) all these restrictions can be satisfied by a proper choice of $\varkappa$.

Summarizing the above discussion, we have shown that for models with $n_{f}=1$ one can identify non-Abelian vortices on Riemann surfaces $\Sigma$ with $\mathrm{SU}(2)$-equivariant instantons on manifolds $\Sigma \times$ $\mathbb{C} P^{1}$. It would be interesting to generalize these results to the case $n_{f}>1$ and arbitrary $n_{c}(=n)$. 


\section{Integrability of non-Abelian BPS vortex equations}

Integrable case. We considered the BPS vortex equations (3.10) on a Riemann surface $\Sigma$ and showed their equivalence to the instanton equations (3.6) on the manifold $M=\Sigma \times \mathbb{C} P^{1}$. Note that one can rescale the metric on $\Sigma, g_{z \bar{z}} \rightarrow t^{2} g_{z \bar{z}}$, so that

$$
R_{\Sigma}^{t}=\frac{2 \varkappa}{t^{2}}=: 2 \varkappa_{t}
$$

and for $g>1$ one can always choose $t^{2}=-R^{2} / \varkappa($ since $\varkappa<0)$ so that

$$
R_{M}=R_{\Sigma}^{t}+R_{\mathbb{C} P^{1}}=0 .
$$

In this case the Weyl tensor for the manifold $M$ is self-dual [16] and such manifolds are considered as gravitational instantons in conformal gravity [20, 15].

An important feature of Kähler manifolds with zero scalar curvature (4.2) is that the so-called twistor space $\mathcal{Z}$ of $M$ becomes a complex manifold if (4.2) is satisfied. Let us consider an open subset $\mathcal{U}$ of $M=\Sigma \times \mathbb{C} P^{1}$ with complex coordinates $z, y$. Then the twistor space ${ }^{4}$ of $\mathcal{U}$ (i.e. the restriction of $\mathcal{Z}$ to $\mathcal{U}$ ) is diffeomorphic to $\mathcal{U} \times \mathbb{C} P^{1}$,

$$
\left.\mathcal{Z}\right|_{\mathcal{U}} \simeq \mathcal{U} \times \mathbb{C} P^{1}
$$

with a local complex coordinate $\lambda \in \mathbb{C} P^{1} \backslash\{\infty\}$ on the last factor. On $\mathcal{Z}$ there is a distribution generated by three vector fields of type $(0,1)$ closed under the Lie bracket. They have the form [12]

$$
V_{\overline{1}}:=\tilde{e}_{\overline{1}}-\lambda \tilde{e}_{2}, \quad V_{\overline{2}}:=\tilde{e}_{\overline{2}}+\lambda \tilde{e}_{1} \quad \text { and } \quad V_{\overline{3}}=\partial_{\bar{\lambda}}
$$

where

$$
\begin{array}{ll}
\tilde{e}_{1}=\rho^{-1}\left(\partial_{z}-\left(\partial_{z} \log \rho\right) \lambda \partial_{\lambda}\right), & \tilde{e}_{\overline{1}}=\rho^{-1}\left(\partial_{\bar{z}}+\left(\partial_{\bar{z}} \log \rho\right) \lambda \partial_{\lambda}\right), \\
\tilde{e}_{2}=\tilde{\rho}^{-1}\left(\partial_{y}-\left(\partial_{y} \log \tilde{\rho}\right) \lambda \partial_{\lambda}\right), & \tilde{e}_{\overline{2}}=\tilde{\rho}^{-1}\left(\partial_{\bar{y}}+\left(\partial_{\bar{y}} \log \tilde{\rho}\right) \lambda \partial_{\lambda}\right) .
\end{array}
$$

Recall that $\rho^{2}=g_{z \bar{z}}$ and $\tilde{\rho}^{2}=g_{y \bar{y}}$ are components of metrics on $\Sigma$ and $\mathbb{C} P^{1}$; the explicit form of $\tilde{\rho}$ is given in (2.4). In the case (4.2) one can pull back the instanton bundle $\mathcal{E} \rightarrow M$ to the bundle $\hat{\mathcal{E}}$ over the twistor space $\mathcal{Z}$ and introduce integrable holomorphic structure on $\hat{\mathcal{E}}$ defined by a (0,1)-type connection along the vector fields (4.4). The integrability of this structure is equivalent $[18,25]$ to the self-duality equations (3.6).

Lax pair. For the case (4.2) from (3.14a) we obtain the inequality

$$
N \leq 2(g-1)
$$

since $\varkappa_{t}=-1 / R^{2}$. For having nonempty moduli space of solutions of the vortex equations $(3.10)$ we assume that this condition is satisfied.

Let us now introduce an $n \times n$ matrix $\psi=\psi\left(x^{\mu}, \lambda\right)$ which does not depend on $\bar{\lambda}$ and consider two linear equations (Lax pair),

$$
\begin{aligned}
& \hat{\nabla}_{V_{\overline{1}}} \psi:=\left[\tilde{e}_{\overline{1}}+\mathcal{A}_{\overline{1}}-\lambda\left(\tilde{e}_{2}+\mathcal{A}_{2}\right)\right] \psi=0, \\
& \hat{\nabla}_{V_{\overline{2}}} \psi:=\left[\lambda\left(\tilde{e}_{1}+\mathcal{A}_{1}\right)+\tilde{e}_{\overline{2}}+\mathcal{A}_{\overline{2}}\right] \psi=0,
\end{aligned}
$$

\footnotetext{
${ }^{4}$ For more details about twistor spaces see e.g. [18].
} 
where $\tilde{e}_{1}, \tilde{e}_{2}, \tilde{e}_{\overline{1}}$ and $\tilde{e}_{\overline{2}}$ are written down in (4.5) and $\mathcal{A}_{1}, \mathcal{A}_{2}, \mathcal{A}_{\overline{1}}$ and $\mathcal{A}_{\overline{2}}$ can be easily extracted from (3.1). It is not difficult to check that the compatibility conditions of the linear equations (4.7),

$$
\left[\hat{\nabla}_{V_{\overline{1}}}, \hat{\nabla}_{V_{\overline{2}}}\right] \psi=0
$$

are equivalent to the vortex equations (3.10). Thus, one can apply various solution-generating techniques (twistor approach, dressing method etc.) for solving non-Abelian BPS vortex equations on $\Sigma$ with the help of the Lax pair (4.7).

To sum up, we have shown that the non-Abelian vortex equations on Riemann surfaces of genus $g>1$ are integrable under certain topological restrictions. It is of interest to generalize the constructions of this paper to supersymmetric vortex equations on $\Sigma$ since they will correspond to solutions of $\mathcal{N}$-extended supersymmetric self-dual Yang-Mills theory $[26,27]$ defined on the conformal gravitational instantons $\Sigma \times S^{2}$. Such vortices on $\Sigma$ will represent the simplest natural interaction of super-Yang-Mills instantons and gravitons in conformal (super)gravity appearing in twistor string theory $[14,15]$. These Yang-Mills/gravity configurations can be a good test background for calculation of open/closed twistor string amplitudes and the corresponding YangMills/gravity amplitudes.

\section{References}

[1] A. A. Abrikosov, Sov. Phys. JETP 5 (1957) 1174 [Zh. Eksp. Teor. Fiz. 32 (1957) 1442];

H. B. Nielsen and P. Olesen, Nucl. Phys. B 61 (1973) 45.

[2] S. B. Bradlow, Commun. Math. Phys. 135 (1990) 1; J. Diff. Geom. 33 (1991) 169;

O. García-Prada, Bull. London Math. Soc. 26 (1994) 88.

[3] N. S. Manton, Nucl. Phys. B 400 (1993) 624;

P. A. Shah and N. S. Manton, J. Math. Phys. 35 (1994) 1171 [hep-th/9307165].

[4] N. S. Manton and S. M. Nasir, Commun. Math. Phys. 199 (1999) 591 [hep-th/9807017];

J. M. Baptista and N. S. Manton, J. Math. Phys. 44 (2003) 3495 [hep-th/0208001].

[5] O. García-Prada, Commun. Math. Phys. 156 (1993) 527; Int. J. Math. 5 (1994) 1.

[6] K. Cieliebak, A. Rita Gaio, I. Mundet i Riera and D. A. Salamon, J. Symplectic Geom. 1 (2002) 543.

[7] A. D. Popov and R. J. Szabo, J. Math. Phys. 47 (2006) 012306 [hep-th/0504025].

[8] O. Lechtenfeld, A. D. Popov and R. J. Szabo, J. High Energy Phys. 09 (2006) 054 [hepth/0603232]; "Quiver gauge theory and noncommutative vortices," arXiv:0706.0979 [hep-th].

[9] A. Hanany and D. Tong, J. High Energy Phys. 07 (2003) 037 [hep-th/0306150];

R. Auzzi, S. Bolognesi, J. Evslin, K. Konishi and A. Yung, Nucl. Phys. B 673 (2003) 187 [hep-th/0307287].

[10] M. Eto, Y. Isozumi, M. Nitta, K. Ohashi and N. Sakai, Phys. Rev. Lett. 96 (2006) 161601 [hep-th/0511088]; M. Eto, K. Hashimoto, G. Marmorini, M. Nitta, K. Ohashi and W. Vinci, Phys. Rev. Lett. 98 (2007) 091602 [hep-th/0609214]. 
[11] D. Tong, "TASI lectures on solitons," hep-th/0509216; M. Eto, Y. Isozumi, M. Nitta, K. Ohashi and N. Sakai, J. Phys. A 39 (2006) R315 [hep-th/0602170]; K. Konishi, "The magnetic monopoles seventy-five years later," hep-th/0702102; M. Shifman and A. Yung, "Supersymmetric solitons and how they help us understand non-abelian gauge theories," hep-th/0703267.

[12] A. D. Popov, "Integrability of vortex equations on Riemann surfaces," arXiv:0712.1756 [hepth].

[13] C. H. Taubes, J. Diff. Geom. 52 (1999) 453.

[14] E. Witten, Commun. Math. Phys. 252 (2004) 189 [hep-th/0312171];

N. Berkovits, Phys. Rev. Lett. 93 (2004) 011601 [hep-th/0402045].

[15] N. Berkovits and E. Witten, J. High Energy Phys. 08 (2004) 009 [hep-th/0406051].

[16] C. P. Boyer, Math. Ann. 274 (1986) 517; C. LeBrun, Proc. Amer. Math. Soc. 98 (1986) 637.

[17] R. Penrose, Gen. Rel. Grav. 7 (1976) 31.

[18] M. F. Atiyah, N. J. Hitchin and I. M. Singer, Proc. Roy. Soc. Lond. A 362 (1978) 425.

[19] A. D. Popov, J. Math. Phys. 46 (2005) 073506 [hep-th/0412042].

[20] G. W. Gibbons and C. N. Pope, Commun. Math. Phys. 61 (1978) 239;

A. Strominger, G. T. Horowitz and M. J. Perry, Nucl. Phys. B 238 (1984) 653.

[21] G. W. Gibbons and M. J. Perry, Nucl. Phys. B 146 (1978) 90;

G. W. Gibbons and S. W. Hawking, Commun. Math. Phys. 66 (1979) 291;

G. W. Gibbons, "Gravitational instantons: a survey," Lecture Notes Phys. 116 (1980) 282.

[22] P. Forgács and N. S. Manton, Commun. Math. Phys. 72 (1980) 15.

[23] C. H. Taubes, Commun. Math. Phys. 75 (1980) 207.

[24] E. B. Bogomolny, Sov. J. Nucl. Phys. 24 (1976) 449.

[25] R. S. Ward, Phys. Lett. A 61 (1977) 81.

[26] A. M. Semikhatov, Phys. Lett. B 120 (1983) 171;

I. V. Volovich, Phys. Lett. B 123 (1983) 329;

W. Siegel, Phys. Rev. D 46 (1992) R3235 [hep-th/9205075].

[27] A. D. Popov and C. Saemann, Adv. Theor. Math. Phys. 9 (2005) 931 [hep-th/0405123];

A. D. Popov, C. Saemann and M. Wolf, J. High Energy Phys. 10 (2005) 058 [hep-th/0505161];

A. D. Popov, Phys. Lett. B 647 (2007) 509 [hep-th/0702106]. 